\title{
Electrostatic versus Resonance Interactions in Photoreceptor Proteins: The Case of Rhodopsin
}

\author{
Riccardo Guareschi, ${ }^{\dagger}$ Omar Valsson ${ }^{\ddagger}$ Carles Curutchet, ${ }^{\complement}$ Benedetta Mennucci, ${ }^{*, \S}$ \\ and Claudia Filippi*, $\dagger$ \\ $\dagger M E S A+$ Institute for Nanotechnology, University of Twente, P.O. Box 217, 7500 AE \\ Enschede, The Netherlands \\ $\ddagger$ Department of Chemistry and Applied Bioscience, ETH Zurich and Facoltà di \\ Informatica, Instituto di Scienze Computazionali, Università della Svizzera italiana, Via \\ Giuseppe Buffi 13, CH-6900, Lugano, Switzerland \\ IDepartament de Fisicoquímica, Facultat de Farmàcia, Universitat de Barcelona, Av. Joan \\ XXIII, s/n 08028 Barcelona, Spain \\ $\S$ Dipartimento di Chimica e Chimica Industriale, Università di Pisa, Via Giuseppe Moruzzi \\ 13, 56124 Pisa, Italy \\ E-mail: benedetta.mennucci@unipi.it; c.filippi@utwente.nl
}




\begin{abstract}
Light-sensing in photoreceptor proteins is subtly modulated by the multiple interactions between the chromophoric unit and its binding pocket. Many theoretical and experimental studies have tried to uncover the fundamental origin of these interactions but reached contradictory conclusions as to whether electrostatics, polarization, or intrinsically quantum effects prevail. Here, we select rhodopsin as prototypical photoreceptor system to reveal the molecular mechanism underlying these interactions and regulating the spectral tuning. Combining a multireference perturbation method and density functional theory with a classical but atomistic and polarizable embedding scheme, we show that accounting for electrostatics alone leads to a qualitatively wrong picture, while a responsive environment can successfully capture both the classical and quantum dominant effects. Several residues are found to tune the excitation both differentially stabilizing ground and excited states, and through non-classical "inductive resonance" interactions. The results obtained with such a quantum-in-classical model are validated against both experimental data and fully quantum calculations.
\end{abstract}

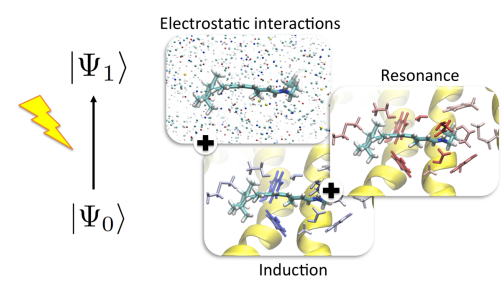

KEYWORDS: Photoreceptor proteins, rhodopsin, multiscale modeling of excited states, QM/MM, polarizable embedding, TDDFT, CASSCF

Photoreceptor proteins play a fundamental role in the function of many biological systems. Specific to every system is how the initial step of light absorption is subtly tuned by the protein matrix which acts quite differently than a more homogeneous environment like a solvent. In particular, the residues closely surrounding the chromophoric unit can significantly affect its response to light but also longer-range effects, well beyond the first shell of interacting residues, are non-negligible. Rationalizing the essence of the regulatory mechanism of absorption is of fundamental importance not only to understand a specific photoreceptor but also to get a more general appreciation of how these light-sensing systems have optimized their structure and molecular composition. To this aim, three precise questions have to be answered: (i) Are the chromophore-protein interactions purely classical (mostly electrostatic and induction) or do we need to account for quantum effects? (ii) Which residues are ac- tive in the excitation process and what is the nature of their contribution? (iii) Can we define a unique theoretical framework for all these interactions?

The best computational strategy to answer these questions might seem to use an expensive quantum chemical description for an extended region beyond the chromophore's boundaries. This would certainly allow us to establish reference target values for excited-state properties at the chosen level of theory but, ultimately, hinder their analysis due to the impossibility of disentangling the real nature of the interactions. A more effective strategy both in terms of computational cost and of ability to further our understanding is represented by a focused approach where the quantum description is limited to the photoexcited site while the embedding protein environment is treated classically but still preserving the atomistic details. Most previous applications of such a strategy to photoreceptor proteins have however employed embedding 
schemes that only account for electrostatic effects, especially through the use of static point charges. These approaches, however, are not only unsuitable to recover the complete essence of the chromophore-protein coupling but are also prone to lead to incorrect interpretations. Here, we demonstrate that atomistic but polarizable classical embeddings can be successfully used to get a correct description of electrostatics and induction interactions and also to account for non-classical effects ${ }^{1}$ as previously found for continuum representations of solvated systems. $^{2,3}$ Moreover, we show that the same analysis can be employed to fingerprint the role of the surrounding residues in determining the excitation of the embedded chromophore.

We focus on a well-known archetype of photoreceptor protein, namely, one of the visual opsins, the bovine rhodopsin. Visual opsins regulate the process of vision at the molecular level and have become a prime playground for the understanding and further manipulation of how a protein environment controls the light-activated response of an embedded photosensitive species. ${ }^{4-6}$ It is remarkable that most members of this large family of receptors share the same photosensitive component, the 11-cis retinal protonated Schiff base (RPSB) chromophore (Fig. 1), and that relatively minor variations in the protein surroundings can tune its absorption over a spectral range as wide as 420-570 nm. This is the result of multiple interactions between the chromophore and the protein whose fundamental origin, however, has so far eluded our understanding.

Bovine rhodopsin, in particular, has been extensively studied both experimentally ${ }^{4,6-8}$ and theoretically. ${ }^{9-25}$ This protein exhibits a strong absorption centered at $2.49 \mathrm{eV}$, dominated by the $\pi \rightarrow \pi^{*}$ transition on the RPSB chromophore, and associated with electronic charge transfer from the $\beta$-ionone ring towards the terminal side of the conjugated chain. From qualitative considerations, one expects that the electrostatic interaction of the positive chromophore with its Glu113 counterion (Fig. 1) induces a blue-shift in absorption with respect to the isolated system. The size of this shift and the role played by the rest of the protein in com-

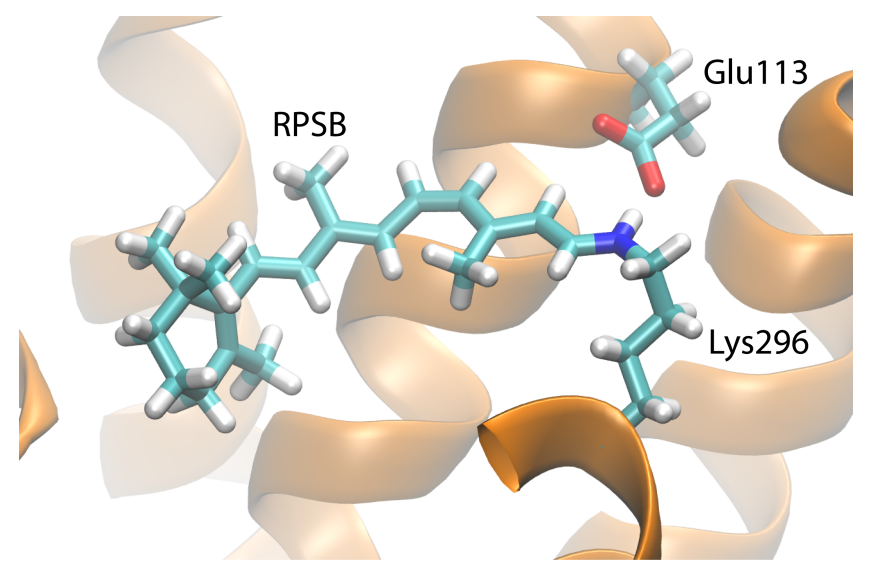

Figure 1: The RPSB chromophore with the Glu113 counterion. We also show the side chain of the Lys296 residue covalently linked to the chromophore.

pensating this shift has been much debated over the years with estimates for both effects often differing considerably in magnitude. ${ }^{9,12-16,19}$

Given the complexity of the system, multiscale approaches to bridge between the smaller scales of the chromophore and the larger protein environment have been commonly employed to study the absorption properties of opsins, in particular, the quantum-mechanicsin-molecular-mechanics (QM/MM) approaches where the environment is treated as a sea of classical static point charges. However, when opting for such a multiscale scheme, one has implicitly acknowledged that the environment has not an active role in the photo-induced process. To overcome these limitations, recent theoretical studies have extended the quantum treatment to larger regions and suggested that at least one shell of amino acids in proximity of the RPSB chromophore participates in the photo-induced process. ${ }^{20,24}$ These findings are qualitatively in line with earlier studies ${ }^{26-28}$ on prototypical opsins by Elstner and coworkers, who found the environment to electrostatically respond to the photoexcitation of the chromophore when relaxed in a state-specific manner using either a quantum-in-quantum partition or through polarizable force fields. In all these cases, the observed effects were classified as "polarization" but is the polarization captured by a responsive (quantum or classical) treatment of the environment the same one re- 
covered in the quantum calculation of a large photoexcited region?

To answer this fundamental question, we begin our analysis of rhodopsin from the TDDFT excitation energies (with the CAM-B3LYP exchange-correlation functional ${ }^{29}$ ) calculated using an electrostatic (QM/MM) or a polarizable embedding (based on atomic charges in combination with induced dipoles in a so-called QM/MMpol approach). ${ }^{30}$ In the latter case, three different schemes are employed to selectively introduce different types of interactions between the RPSB chromophore and the protein. First, we polarize the dipoles to the ground-state density of RPSB and keep them frozen in the calculation of the excitation energy (polGS). Then, state-specific dipoles are used to account for possible differential electrostatic and induction effects between ground and excited states (polSS). Given the large transfer of electronic charge following photoexcitation, one would expect a strong electrostatic interaction of RPSB with its surrounding and a sizable polSS correction on the polGS excitation energies (a rather small shift, however, was previously reported for one structural realization of the system ${ }^{27}$ ). In the last scheme (polRes), we adopt a computational framework where the induced dipoles respond to the transition density of the photoexcited species. This embedding condition accounts for the non-classical part of the coupling between the chromophore excitation and the environment which, in previous analyses within a continuum formulation of the classical subsystem, has been described as a dispersion-like term, ${ }^{2}$ an excitonic coupling, ${ }^{3}$ or an "inductive resonance". ${ }^{31}$ This last definition will be adopted also in the present study.

To account for temperature effects and avoid the risk of limiting the analysis to a single and possibly not representative structure, we consider here different sets of frames extracted from QM/MM molecular dynamics (MD) trajectories where the QM part is treated within DFT using the PBE exchange-correlation functional $^{32}$ (see SI for the details). Frames 1-6 are the central frames of the most populated clusters obtained in a cluster analysis of a trajectory we generated with the chromophore and

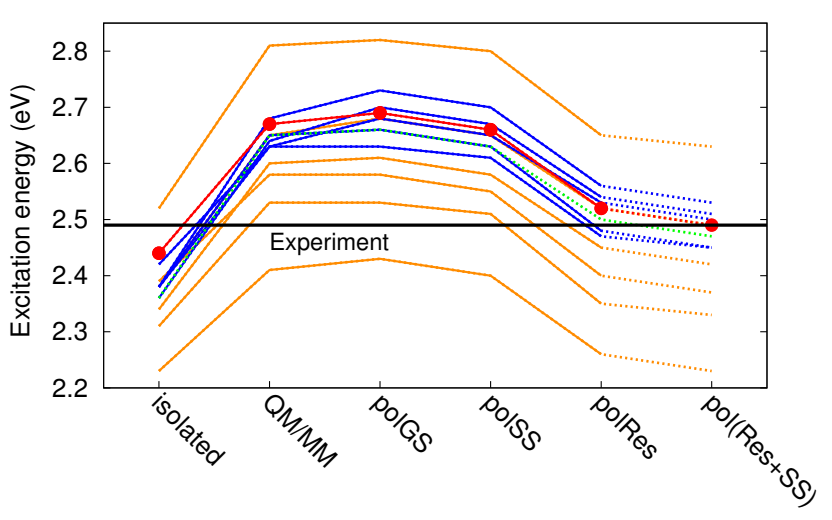

Figure 2: TDDFT excitation energies (eV) computed for RPSB isolated and in a rhodopsin environment described with static point charges (QM/MM) and with dipoles induced in the ground state (polGS), in a state-specific (polSS), and in a resonant formulation (polRes). The orange lines identify the frames (1-6) from cluster analysis, the blue and green lines the frames from Ref. ${ }^{20}$ (frames $7-12$ ), and the red circles an annealed frame. The experimental absorption maximum is at $2.49 \mathrm{eV}$.

the Glu113 counterion in the QM region. We then analyze a second set (frames 7-11) obtained in a previous study ${ }^{20}$ from a different QM/MM trajectory and selected from the region of phase space which gives excitation energies close to the maximum of the theoretical absorption band computed at the ZINDO level. Finally, we include two more structures, an annealed structure from the same trajectory of frames 1-6 and frame 12 from another trajectory previously obtained with also the anionic residue Glu181 included in the $\mathrm{QM}$ region ${ }^{20}$ and selected with the same recipe used to obtain frames $7-11$. All the results are summarized in Fig. 2.

The excitation energies of all snapshots follow precisely the same trend: the inclusion of static point charges causes a significant blue shift of the excitation energy (about $0.2 \mathrm{eV}$ ) with respect to the values computed on the isolated chromophore at the geometry extracted from the protein. This shift can be mostly attributed to the presence of the counterion stabilizing the ground state and inhibiting the charge transfer in the excited state. A very similar shift is ob- 
tained when the chromophore is embedded in an environment with its polarization being kept frozen to the ground state of RPSB (polGS): the excitation energies are only somewhat more blue-shifted than the MM values as a consequence of the further stabilization of the ground state. The effect of this improved groundstate description is however rather small at the TDDFT level, being at most $0.06 \mathrm{eV}$ and in average $0.02 \mathrm{eV}$. If we further enhance the description of the chromophore-protein coupling by allowing the induced dipoles to relax according to the excited state (polSS), we recover a red-shift in the excitation energies with respect to the polGS values, which is however rather small and consistently between -0.03 and $-0.02 \mathrm{eV}$. The presence of a responsive environment has a much larger effect only when the induced dipoles are calculated within the resonance regime (polRes), namely, in response to the transition density associated with the electronic excitation. The non-classical part of the coupling captured in this model appears therefore to play a dominant role, lowering the excitation energies by as much as $0.16-0.18 \mathrm{eV}$ with respect to the polGS value and largely recovering the so-called counterion quenching which was lost in an electrostatic treatment of the protein. We finally note that the trend of excitation energies with respect to the different models of the environment is similar in all frames though the latter are selected based on very different criteria. In particular, while frames $7-11$ can be considered as biased towards the close neighborhood of the chromophores as they have been selected on the basis of the excitation energies, the choice of frames (1-6) has been based on a cluster analysis performed over the complete protein and, consequently, they are expected to yield a larger spread of excitation energies (see SI for further discussion).

To better understand the origin of the calculated shifts we recall that, perturbatively to linear order, the polSS and polRes corrections with respect to the polGS excitation energies are proportional to the square of the variation of the molecular dipole between ground and excited states, and to the transition dipole mo- ment, respectively, so that ${ }^{33}$

$$
\frac{\Delta E_{\text {exc }}^{(\mathrm{SS}-\mathrm{GS})}}{\Delta E_{\mathrm{exc}}^{(\mathrm{Res}-\mathrm{GS})}} \approx \frac{|\Delta \boldsymbol{\mu}|^{2}}{2\left|\boldsymbol{\mu}_{01}\right|^{2}},
$$

with $\Delta E_{\mathrm{exc}}^{(\mathrm{XX}-\mathrm{GS})}=E_{\mathrm{exc}}^{\mathrm{polXX}}-E_{\mathrm{exc}}^{\mathrm{polGS}}$. If we first focus on the state-specific contribution, we observe that we would have expected a more sizable reduction of the excitation energy due to the inclusion of differential polarization effects: the excited state of RPSB displays a strong charge redistribution from the $\beta$-ionone ring to the nitrogen terminus which should be greatly stabilized by the relative optimization of the chromophore surroundings. The difference between the ground- and the excited-state molecular dipole moment is in fact experimentally rather large (ca. 12-13 Debye). ${ }^{34,35}$ The underestimation of this electrostatic differential contribution can be explained as resulting from a limitation of TDDFT/CAM-B3LYP, which tends to underestimate the dipole moment changes in charge-transfer excitations. ${ }^{36}$ Our calculations for rhodopsin are affected by the same problem and yield values of $|\Delta \boldsymbol{\mu}|$ (computed in polGS embedding) that are too low by about a factor of two. This incorrectly predicted change in the dipole moment translates in an underestimation by about a factor of four of the electrostatic differential polarization contributions in the TDDFT excitation energies as further discussed below in comparison with wavefunction-based calculations.

The polRes corrections to the polGS excitation energies are instead proportional to the magnitude square of the transition dipole moment which, for the bright $\pi \rightarrow \pi^{*}$ transition of RPSB in rhodopsin, is quite large. The TDDFT transition dipole moments of about 12 Debye (see Table 1) lead in fact to sizable polRes contributions to the excitation energies. In this case, the estimates can be considered reliable as TDDFT/CAM-B3LYP has generally been shown to produce reasonably good oscillator strengths. ${ }^{37}$

The polRes and polSS schemes account therefore for different components of the chromophore-protein interaction that co-exist in rhodopsin. We can thus extrapolate the 
combined effects of these polarization treatments by adding the two corrections on the polGS excitation energies as shown in Figure 2. As discussed below, such an additive approach appears to be appropriate for rhodopsin, being supported by the comparison with TDDFT calculations on large clusters. The extrapolated excitation energies are reduced by about $0.2 \mathrm{eV}$ with respect to the polGS values and, for the snapshots energetically close to the annealed one, in good agreement with the experimental absorption maximum. Nevertheless, we should not attribute too much significance to this agreement: We must recall that the state-specific contribution is underestimated in TDDFT, resulting in a blue-shift which is fortuitously compensated by the use of an RPSB geometry computed with a generalized gradient approximation, yielding a smaller bond length alternation and a consequent red-shift. Furthermore, we are neglecting vibronic effects which are not negligible in photoactive rhodopsin and should result in the computed vertical excitation energy being higher than the absorption maximum.

The TDDFT/MMpol calculations allows us to further deepen our analysis and identify which residues respond more strongly and whether they polarize to the ground state, the excited state or in a resonance fashion. In Figure 3, we show for the annealed frame the dominant contributions to the ground-state polarization $\left(\mathrm{E}_{\mathrm{pol}}^{\mathrm{GS}}\right)$ and the contributions induced at the polSS and polRes level relative to polGS:

$$
\delta \mathrm{E}_{\mathrm{pol}}^{\mathrm{XX}}=-\frac{1}{2} \sum_{a} \boldsymbol{\mu}_{a}\left(\rho^{\mathrm{XX}}\right) \cdot \mathbf{E}_{a}\left(\rho^{\mathrm{XX}}\right)
$$

where the sum is over the classical polarizable sites and the dipoles are induced either by the transition density, $\rho^{\text {Res }}=\rho_{01}$, or by the density difference, $\rho^{\mathrm{SS}}=\Delta \rho$, between the ground and excited states of RPSB. For a set of frames, we then determine the residues with contributions to $\delta \mathrm{E}_{\text {pol }}$ larger than about 0.09 $\mathrm{kcal} / \mathrm{mol}$. Since the polSS correction to polGS is underestimated by about a factor of 4 as discussed above, we identify the residues whose polSS contributions rescaled by the same fac-

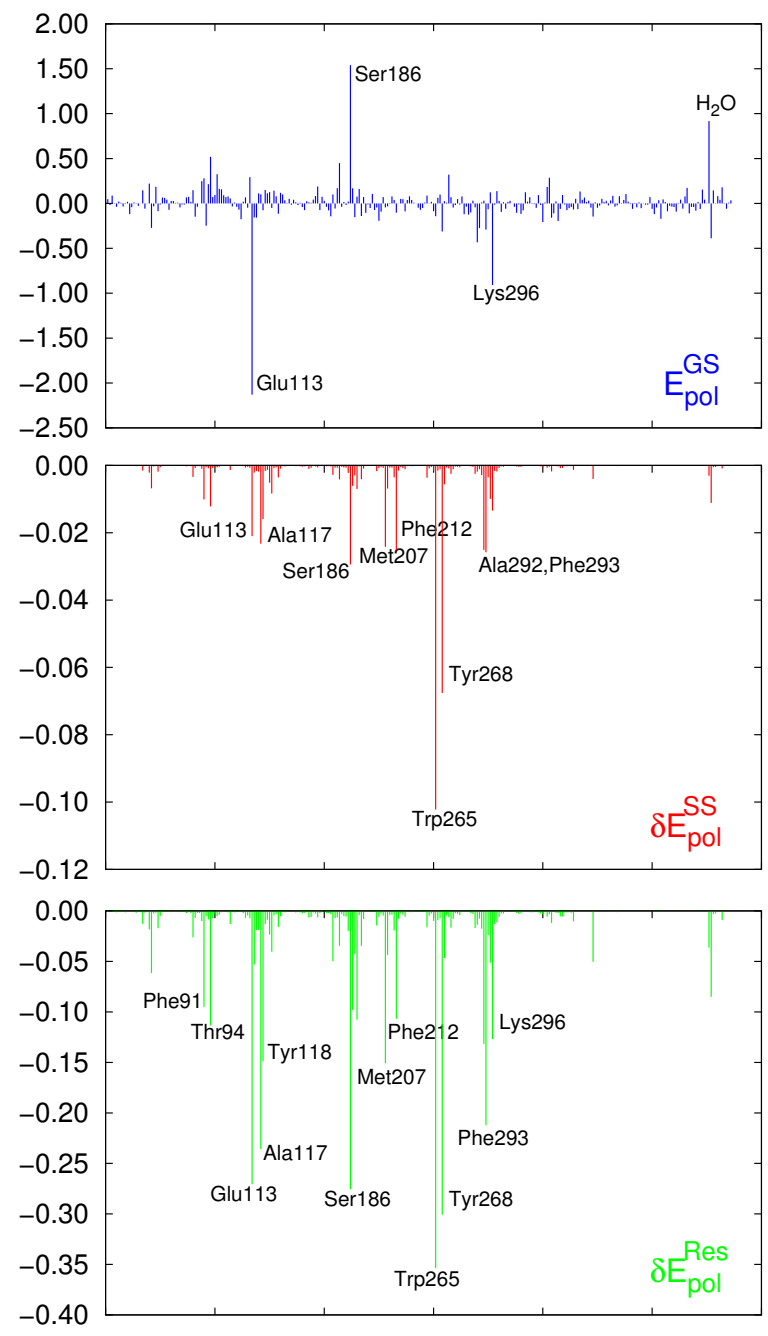

Figure 3: Contributions (kcal $/ \mathrm{mol}$ ) to the ground-state DFT polarization energy $\left(\mathrm{E}_{\mathrm{pol}}^{\mathrm{GS}}\right)$ of all residues within $15 \AA$ of RPSB (top) and relative TDDFT contributions $\left(\delta \mathrm{E}_{\mathrm{pol}}\right)$ computed within the polSS (middle) and polRes (bottom) schemes for the annealed frame.

tor is above the chosen threshold. In Fig. 4, we show the position of the common set of surviving residues, which nicely embrace the RPSB chromophore.

It is first important to appreciate that the dominating contributions to the ground-state polarization are mostly carried by few residues in proximity of the nitrogen end of RPSB, in particular, the covalently linked Lys296, the counterion Glu113, a water molecule occasionally hydrogen-bonded to it, and Ser186, while the response to the excitation are spread over a larger number of residues surrounding also the $\beta$-ionone ring. As expected, the polSS 
a)

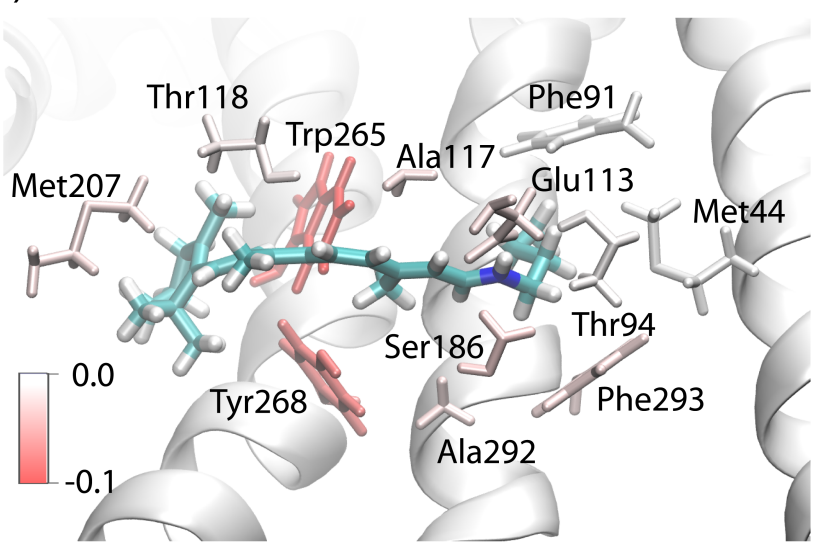

b)

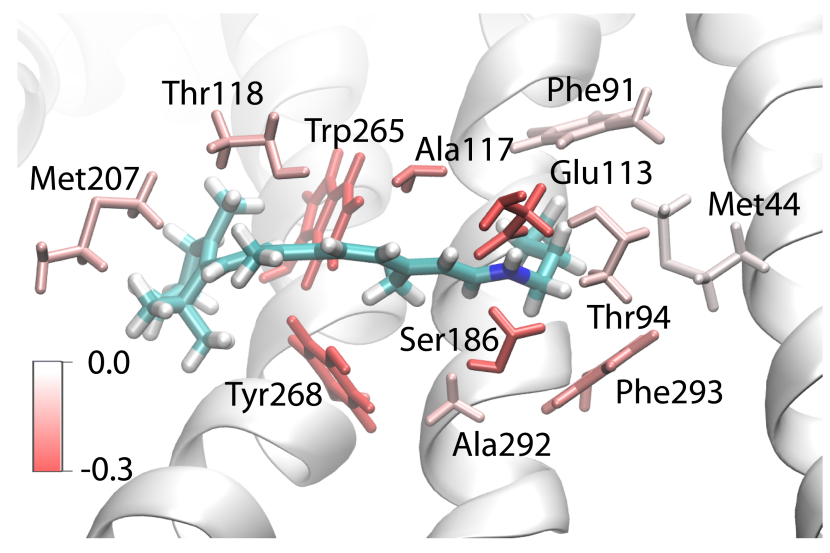

Figure 4: The RPSB chromophore covalently linked to Lys296 and the residues with the largest contributions $\delta \mathrm{E}_{\text {pol }}$ to the relative polarization energies in the a) polSS and b) polRes schemes. The residues are visualized up to their $\mathrm{C}_{\alpha}$ atoms.

and polRes responses correlate rather well: the residues more strongly interacting with the chromophore in the excited state are also the ones that predominantly interact at the polRes level. In addition to the three obvious candidates close to the positive terminus of RPSB (Lys296, Glu113, Ser186), the residues displaying a sizable $\pi$ electronic system (Trp265, Tyr268, Phe293) contribute the most both to the polSS and polRes responses, with Trp265 $\pi$-stacked to one end of the chromophore giving the absolute largest value in several frames. A few residues close to the central part of the conjugated skeleton of the chromophore (Ala117, Tyr118) further stabilize the excitation. We finally note that the validity of this analysis, based on Eq. 2, to pinpoint the role of the var- ious residues is strongly supported by the close equality recovered between the total $\delta \mathrm{E}_{\text {pol }}^{\text {Res }}$ and the excitation energy difference $\Delta \mathrm{E}_{\text {exc }}^{\text {Res-GS }}$ (see SI).

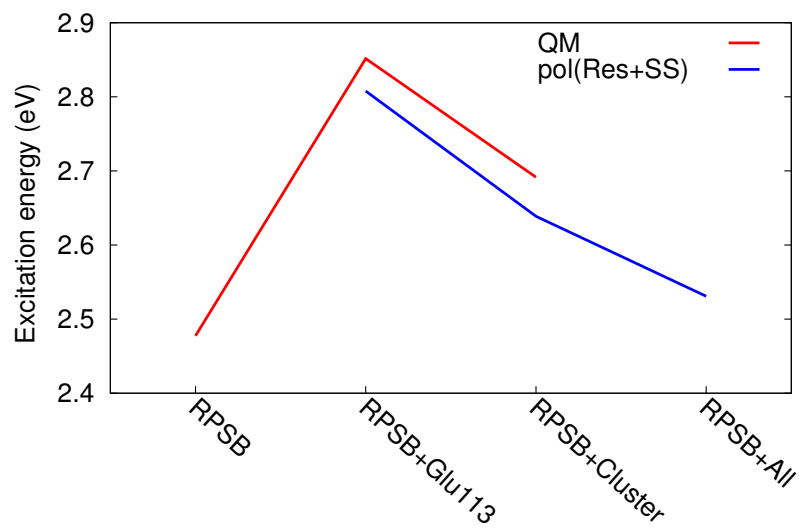

Figure 5: Comparison of the excitation energies $(\mathrm{eV})$ computed for the annealed frame with TDDFT/CAM-B3LYP in a supermolecular calculation on the complete system or within QM/MMpol with only RPSB in the QM region. We consider the isolated chromophore and add the counterion, a cluster of residues (see text), and the whole protein.

We can also exploit this analysis to corroborate our approach and perform supermolecular TDDFT reference calculations on realistic cluster models of the system constructed from the most responsive residues we have identified above. We follow such a procedure for the annealed frame and build two clusters comprising the RPSB chromophore and either the counterion only or all important residues. As shown in Fig. 5, the resulting supermolecular TDDFT excitation energies are in very good agreement with the pol(Res+SS) values, differing by roughly the same small amount and further supporting the validity of our approach. Moreover, this analysis further details the role of the different residues and their influence on the chromophore: the introduction of the nearby Glu113 counterion leads to a large blueshift as the electrostatics is there dominant, stabilizing the charged ground state. By enlarging the number of interacting residues through the cluster, an opposite trend is observed showing that their presence is necessary to allow the sta- 
bilization of the excited state. This stabilization is further enhanced when the full system is accounted for within the MMPol approach.

The understanding gained so far appears to be sound (and internally consistent) within the limitations of TDDFT, which we already know, for instance, to underestimate differential polarization effects. To further validate our observations, we also employ a wavefunctionbased description of the QM region and select three frames $(4,8$, and 12$)$ to be treated with the complete-active-space self-consistent-field (CASSCF) approach followed by second-order perturbation theory (CASPT2). The comparison between the CASPT2 and TDDFT excitation energies is shown in Fig. 6 for the three selected frames and summarized in Table 1. The MM dipoles are induced in a CASSCF calculation targeting either the ground or the excited state, and used unchanged in the PT2 computation of the polGS and polSS excitation energies.

The dependence of the CASPT2 excitation energies on the choice of electrostatic model for the environment follows a similar trend to the one observed at the TDDFT level but the magnitude of the shifts induced by the different descriptions of the environment is much larger. The use of a ground-state-polarized protein raises the excitation energies with respect to point-charge embedding by more than $0.1 \mathrm{eV}$ and accounting for differential polarization effects in the polSS scheme yields a correction of the same magnitude and opposite sign. The change in the correlated excitation energies computed with state-specific dipoles with respect to the polGS values is much larger than in the TDDFT case as a consequence of the more accurate estimation of $|\Delta \boldsymbol{\mu}|$ at the CASPT2 level. In fact, the CASPT2 differences $\Delta \mathrm{E}_{\mathrm{exc}}^{(\mathrm{SS}-\mathrm{GS})}$ can be remarkably well estimated by simply rescaling the corresponding TDDFT values by the ratio squared between the CASPT2 and TDDFT $|\Delta \boldsymbol{\mu}|$, again supporting the validity of our theoretical framework of analysis based on the magnitude of the transition dipole moment and the molecular dipole difference, and the resulting polarization effects. Accidentally, for rhodopsin, the underestimated polGS and polSS responses within TDDFT and the near cancellation of the larger polGS and polSS shifts within CASPT2 yield excitation energies at both levels of theory that are not too dissimilar from the corresponding QM/MM values.

Within CASPT2, we do not have direct access to the excitation energies computed within the resonance formalism but we can estimate them following Eq. 1 and rescaling the CASPT2 polSS corrections with the ratio squared of the CASPT2 transition dipole moment and the variation of the molecular dipoles obtained in a polGS calculation. These estimates are in very good agreement with the corresponding TDDFT linear-response corrections as shown for the three frames in Table 1, where the CASPT2 resonance contributions consistently obtained as in Eq. 1 are listed.

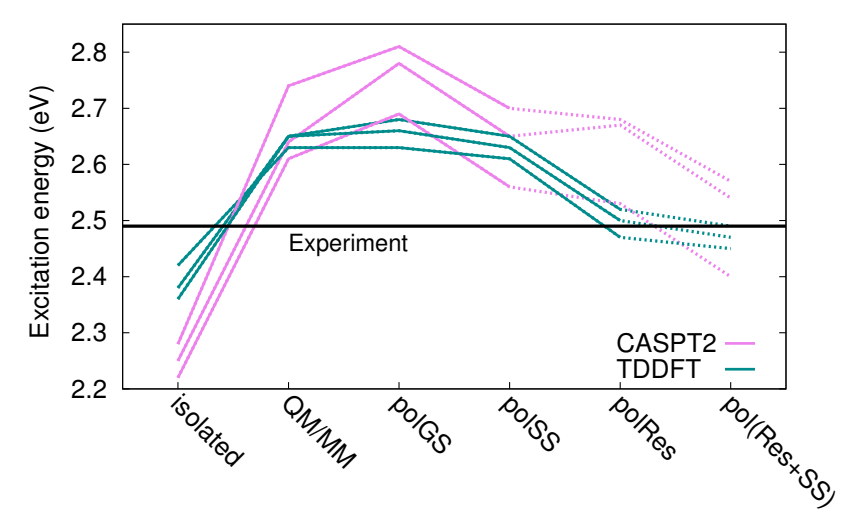

Figure 6: Comparison of the TDDFT/CAMB3LYP and CASPT2 excitation energies (eV) for frames 4, 8, and 12 for RPSB isolated and with different models of the protein environment.

The analysis carried out within TDDFT and CASPT2 reveals a qualitatively similar behavior. Furthermore, the quantitative differences can be rationalized if one accounts for the underestimated electrostatic response of TDDFT in the excited state. From both analyses, it is clear that an embedding scheme based on fixed point charges is not only too simplistic to capture the complexity of the protein-chromophore interactions but it also can lead to qualitatively wrong results. The comparison with the different polarizable models has here given us the needed clues on where a point-charge model goes amiss. We have also seen that, unfor- 
Table 1: Excitation energies (eV) of rhodopsin computed with nonpolarizable QM/MM and the QM/polGS scheme using TDDFT/CAM-B3LYP and CASPT2/ANO-L-VDZP. The polSS and polRes corrections to the polGS values and the extrapolated excitation energies are also listed. The polRes results are computed at the TDDFT level and estimated in CASPT2 by rescaling the CASPT2 polSS corrections according to Eq. 1. The polGS transition dipole moment, $\left|\mu_{01}\right|$, and the variation of the molecular dipole moment, $|\Delta \mu|$, are given in Debye.

\begin{tabular}{|c|c|c|c|c|c|c|c|}
\hline Frame & $\left|\boldsymbol{\mu}_{01}\right|$ & $|\Delta \mu|$ & $\mathrm{E}_{\mathrm{exc}}^{\mathrm{QM} / \mathrm{MM}}$ & $\mathrm{E}_{\mathrm{exc}}^{\mathrm{polGS}}$ & $\Delta \mathrm{E}_{\mathrm{exc}}^{(\mathrm{SS}-\mathrm{GS})}$ & $\Delta \mathrm{E}_{\mathrm{exc}}^{(\mathrm{Res}-\mathrm{GS})}$ & $\mathrm{E}_{\mathrm{exc}}^{\mathrm{pol}(\operatorname{Res}+\mathrm{SS})}$ \\
\hline \multicolumn{8}{|c|}{ TDDFT } \\
\hline 4 & 11.84 & 8.29 & 2.652 & 2.680 & -0.034 & -0.160 & 2.486 \\
\hline 8 & 11.88 & 5.73 & 2.630 & 2.627 & -0.018 & -0.158 & 2.451 \\
\hline 12 & 11.85 & 7.18 & 2.653 & 2.662 & -0.028 & -0.160 & 2.475 \\
\hline \multicolumn{8}{|c|}{ CASPT2 } \\
\hline 4 & 9.35 & 14.68 & 2.645 & 2.784 & -0.136 & -0.110 & 2.537 \\
\hline 8 & 10.64 & 13.50 & 2.609 & 2.686 & -0.129 & -0.160 & 2.397 \\
\hline 12 & 11.38 & 15.41 & 2.724 & 2.809 & -0.113 & -0.124 & 2.571 \\
\hline
\end{tabular}

tunately, the use of a polarizable embedding frozen in the ground-state does not ameliorate the point-charge results but further blue-shifts the excitation energy. An improved description of ground-state rhodopsin alone is therefore not sufficient and the lack of environmental response to the electronic transition lies at the heart of the problem. The correction due to the relaxation of the protein polarization in the excited state is in fact expected to be large due to the density difference upon excitation resulting from the flux of electronic charge from the $\beta$ ionone ring to the nitrogen terminus of RPSB. When this physical feature is properly reproduced by the selected QM method, as in the case of CASPT2, the state-specific correction gives indeed a red-shift of more than $0.1 \mathrm{eV}$, which can be ascribed to the response of several residues in the protein pocket of the chromophore. Nevertheless, the inclusion of differential polarization effects does not account for the complete description of the chromophoreprotein coupling. The same residues which more strongly polarize in the excited state of the chromophore also "resonate" to the transition density when treated within a linearresponse scheme at the TDDFT level. The resulting correction to the excitation energies is of the same order of magnitude as the statespecific one if the QM method describes equally well the transition density and the change in the density going from the ground to the excited state. If these two contributions are finally added together, the resulting excitation energy is in good agreement with the measured value.

Our analysis on this prototypical photosensitive system demonstrates that the protein tuning of the excitation process is not only of electrostatic nature and is therefore not straightforwardly amenable to a description in terms of a multiscale quantum-in-classical approach. Consequently, the use of static point charges commonly adopted to treat photoreceptor proteins is generally bound to be inadequate. For the same reasons, also a quantum-in-quantum approach based on the product state of chromophore and protein wave functions is expected to fail. The process of light absorption is in fact modulated by the concurrent presence of induction and resonance effects. A computational option to recover them at once is to perform an explicit quantum calculation on an extended region surrounding the photoactive moiety, employing a technique able to describe coupled excitations between the chromophore and the many residues we have shown to participate in the excitation process. ${ }^{38}$ This is unfortunately very expensive and currently limited to the use of low-correlation approaches. Here, we have however outlined an alternative, practical route where a classical embedding model is adapted 
to mimic both induction and resonant coupling, and to provide key information on the origin of the interactions and the detailed quantitative role of specific amino acids in the photoexcitation of a complex chromophore-protein system. Importantly, the subtle interplay between electrostatic and resonance interactions we unveiled in absorption is expected to modulate also subsequent photo-induced processes which often characterize photoreceptor proteins and ultimately regulate their function. The computational framework presented here has the potential to capture the essence of the chromophoreprotein coupling in all relevant steps of their photochemical evolution.

\section{Supporting Information Available:} Computational details; TDDFT, CASSCF, and CASPT2 excitation energies; further analysis of resonance CASPT2 and TDDFT corrections; dominant contributions to polarization energies for selected frames; dependence of TDDFT excitation energies on structural parameters. This material is available free of charge via the Internet at http://pubs.acs.org/.

Acknowledgement R.G. is supported by an ECHO grant (712.012.005) of the Netherlands Organisation for Scientific Research (NWO), and we received support from NWO for the use of the SARA supercomputer facilities. B.M. acknowledges the European Research Council (ERC) in the framework of the Starting Grant (EnLight-277755) and the University of Pisa under the project PRA-2016-46 for financial support.

\section{References}

(1) Daday, C.; Curutchet, C.; Sinicropi, A.; Mennucci, B.; Filippi, C. ChromophoreProtein Coupling beyond Nonpolarizable Models: Understanding Absorption in Green Fluorescent Protein. J. Chem. Theory Comput. 2015, 11, 4825-4839.

(2) Corni, S.; Cammi, R.; Mennucci, B.; Tomasi, J. Electronic Excitation Energies of Molecules in Solution within Continuum Solvation Models: Investigating the Discrepancy between State-Specific and Linear-Response Methods. J. Chem. Phys. 2005, 123, 134512.

(3) Lunkenheimer, B.; Köhn, A. Solvent Effects on Electronically Excited States Using the Conductor-Like Screening Model and the Second-Order Correlated Method $\operatorname{ADC}(2)$. J. Chem. Theory Comput. 2013, 9, 977-994.

(4) Ebrey, T.; Koutalos, Y. Vertebrate Photoreceptors. Prog. Retin. Eye Res. 2001, 20, 49-94.

(5) Nickle, B.; Robinson, P. R. The Opsins of the Vertebrate Retina: Insights from Structural, Biochemical, and Evolutionary Studies. Cell. Mol. Life Sci. 2007, 64, 2917-2932.

(6) Ernst, O. P.; Lodowski, D. T.; Elstner, M.; Hegemann, P.; Brown, L. S.; Kandori, H. Microbial and Animal Rhodopsins: Structures, Functions, and Molecular Mechanisms. Chem. Rev. 2014, 114, 126-163.

(7) Palczewski, K. G Protein-Coupled Receptor Rhodopsin. Annu. Rev. Biochem. 2006, 75, 743-767.

(8) Katayama, K.; Sekharan, S.; Sudo, Y. In Optogenetics: Light-Sensing Proteins and Their Applications; Yawo, H., Kandori, H., Koizumi, A., Eds.; Springer Japan: Tokyo, 2015; Chapter Color Tuning in Retinylidene Proteins, pp 89-107.

(9) Coto, P. B.; Strambi, A.; Ferré, N.; Olivucci, M. The Color of Rhodopsins at the Ab Initio Multiconfigurational Perturbation Theory Resolution. Proc. Natl. Acad. Sci. USA 2006, 103, 17154-17159.

(10) Matsuura, A.; Sato, H.; Houjou, H.; Saito, S.; Hayashi, T.; Sakurai, M. Accurate Evaluation of the Absorption Maxima of Retinal Proteins Based on a $\mathrm{Hy}-$ brid QM/MM Method. J. Comput. Chem. 2006, 27, 1623-1630. 
(11) Hoffmann, M.; Wanko, M.; Strodel, P.; König, P. H.; Frauenheim, T.; Schulten, K.; Thiel, W.; Tajkhorshid, E.; Elstner, M. Color Tuning in Rhodopsins: The Mechanism for the Spectral Shift between Bacteriorhodopsin and Sensory Rhodopsin II. J. Am. Chem. Soc. 2006, 128, 10808-10818.

(12) Fujimoto, K.; Hayashi, S.; Hasegawa, J.; Nakatsuji, H. Theoretical Studies on the Color-Tuning Mechanism in Retinal Proteins. J. Chem. Theory Comput. 2007, 3, 605-618.

(13) Bravaya, K.; Bochenkova, A.; Granovsky, A.; Nemukhin, A. An Opsin Shift in Rhodopsin: Retinal S0-S1 Excitation in Protein, in Solution, and in the Gas Phase. J. Am. Chem. Soc. 2007, 129, 1303513042 .

(14) Altun, A.; Yokoyama, S.; Morokuma, K. Mechanism of Spectral Tuning Going from Retinal in Vacuo to Bovine Rhodopsin and its Mutants: Multireference ab Initio Quantum Mechanics/Molecular Mechanics Studies. J. Phys. Chem. B 2008, 112, 16883-16890.

(15) Andruniów, T.; Olivucci, M. How Does the Relocation of Internal Water Affect Resonance Raman Spectra of Rhodopsin? An Insight from CASSCF/Amber Calculations. J. Chem. Theory Comput. 2009, 5, 3096-3104.

(16) Tomasello, G.; Olaso-González, G.; Altoè, P.; Stenta, M.; Serrano-Andrés, L.; Merchán, M.; Orlandi, G.; Bottoni, A.; Garavelli, M. Electrostatic Control of the Photoisomerization Efficiency and Optical Properties in Visual Pigments: On the Role of Counterion Quenching. $J$. Am. Chem. Soc. 2009, 131, 5172-5186.

(17) Rostov, I. V.; Amos, R. D.; Kobayashi, R.; Scalmani, G.; Frisch, M. J. Studies of the Ground and Excited-State Surfaces of the Retinal Chromophore using CAM-B3LYP. J. Phys. Chem. B 2010, 114, 5547-5555.
(18) Hasegawa, J.; Fujimoto, K. J.; Nakatsuji, H. Color Tuning in Photofunctional Proteins. ChemPhysChem 2011, 12, 3106-3115.

(19) Sekharan, S.; Morokuma, K. Why 11-cisRetinal? Why Not 7-cis-, 9-cis-, or 13-cisRetinal in the Eye? J. Am. Chem. Soc. 2011, 133, 19052-19055.

(20) Valsson, O.; Campomanes, P.; Tavernelli, I.; Rothlisberger, U.; Filippi, C. Rhodopsin Absorption from First Principles: Bypassing Common Pitfalls. J. Chem. Theory Comput. 2013, 9, 24412454 .

(21) Campomanes, P.; Neri, M.; Horta, B. A. C.; Röhrig, U. F.; Vanni, S.; Tavernelli, I.; Rothlisberger, U. Origin of the Spectral Shifts among the Early Intermediates of the Rhodopsin Photocycle. $J$. Am. Chem. Soc. 2014, 136, 3842-3851.

(22) Zhou, X.; Sundholm, D.; Wesołowski, T. A.; Kaila, V. R. I. Spectral Tuning of Rhodopsin and Visual Cone Pigments. J. Am. Chem. Soc. 2014, 136, 2723-2726.

(23) Cheng, C.; Kamiya, M.; Uchida, Y.; Hayashi, S. Molecular Mechanism of Wide Photoabsorption Spectral Shifts of Color Variants of Human Cellular Retinol Binding Protein II. J. Am. Chem. Soc. 2015, 137, 13362-13370.

(24) Suomivuori, C.-M.; Lang, L.; Sundholm, D.; Gamiz-Hernandez, A. P.; Kaila, V. R. I. Tuning the Protein-Induced Absorption Shifts of Retinal in Engineered Rhodopsin Mimics. Chem. Eur. J. 2016, 22, 8254-8261.

(25) Melaccio, F.; Calimet, N.; Schapiro, I.; Valentini, A.; Cecchini, M.; Olivucci, M. Space and Time Evolution of the Electrostatic Potential During the Activation of a Visual Pigment. J. Phys. Chem. Lett. 2016, 7, 2563-2567. 
(26) Wanko, M.; Hoffmann, M.; Frauenheim, T.; Elstner, M. Effect of Polarization on the Opsin Shift in Rhodopsins. 1. A Combined QM/QM/MM Model for Bacteriorhodopsin and Pharaonis Sensory Rhodopsin II. J. Phys. Chem. B 2008, 112, 11462-11467.

(27) Wanko, M.; Hoffmann, M.; Frähmcke, J.; Frauenheim, T.; Elstner, M. Effect of Polarization on the Opsin Shift in Rhodopsins. 2. Empirical Polarization Models for Proteins. J. Phys. Chem. B 2008, 112, 11468-11478.

(28) Welke, K.; Frähmcke, J. S.; Watanabe, H. C.; Hegemann, P.; Elstner, M. Color Tuning in Binding Pocket Models of the Chlamydomonas-Type Channelrhodopsins. J. Phys. Chem. B 2011, 115, 15119-15128.

(29) Yanai, T.; Tew, D. P.; Handy, N. C. A New Hybrid Exchange Correlation Functional Using the Coulomb-Attenuating Method (CAM-B3LYP). Chem. Phys. Lett. 2004, 393, 51-57.

(30) Curutchet, C.; Muñoz-Losa, A.; Monti, S.; Kongsted, J.; Scholes, G. D.; Mennucci, B. Electronic Energy Transfer in Condensed Phase Studied by a Polarizable QM/MM Model. J. Chem. Theory Comput. 2009, 5, 1838-1848.

(31) Cupellini, L.; Amovilli, C.; Mennucci, B. Electronic Excitations in Nonpolar Solvents: Can the Polarizable Continuum Model Accurately Reproduce Solvent Effects? J. Phys. Chem. B 2015, 119, 8984 8991.

(32) Perdew, J. P.; Burke, K.; Ernzerhof, M. Generalized Gradient Approximation Made Simple. Phys. Rev. Lett. 1996, 7r7, 3865-3868.

(33) Cammi, R.; Corni, S.; Mennucci, B.; Tomasi, J. Electronic Excitation Energies of Molecules in Solution: State Specific and Linear Response Methods for Nonequilibrium continuum Solvation Models. J. Chem. Phys. 2005, 122, 104513.

(34) Mathies, R.; Stryer, L. Retinal has a Highly Dipolar Vertically Excited Singlet State: Implications for Vision. Proc. Natl. Acad. Sci. USA 1976, 73, 2169-2173.

(35) Ponder, M.; Mathies, R. Excited-state polarizabilities and dipole moments of diphenylpolyenes and retinal. J. Phys. Chem. 1983, 87, 5090-5098.

(36) Bednarska, J.; Roztoczyńska, A.; Bartkowiak, W.; Zaleśny, R. Comparative assessment of density functionals for excited-state dipole moments. Chem. Phys. Lett. 2013, 584, 58-62.

(37) Caricato, M.; Trucks, G. W.; Frisch, M. J.; Wiberg, K. B. Oscillator Strength: How Does TDDFT Compare to EOM-CCSD? J. Chem. Theory Comput. 2011, 7, 456466.

(38) Neugebauer, J.; Curutchet, C.; MuñozLosa, A.; Mennucci, B. A Subsystem TDDFT Approach for Solvent Screening Effects on Excitation Energy Transfer Couplings. J. Chem. Theory Comput. 2010, 6, 1843-1851. 